\title{
ON LINDELÖF LATTICES AND SEPARATION
}

\author{
GEORGE M. EID \\ Department of Mathematics \\ John Jay College of Criminal Justice \\ The City University of New York \\ 445 West 59th Street \\ New York, NY 10019 U.S.A. \\ (Received June 29, 1990 and in revised form October 17, 1990)

\begin{abstract}
Let $X$ be an abstract set and $\mathcal{L}$ a lattice of subsets of $X$. Some general properties of Lindelöf, regular as well as normal lattices are investigated for their measure implications and their relationship to separation properties. Moreover, we show that the generalized Wallman replete space and the generalized Wallman prime complete space are Lindelöf spaces if and only if certain measure relationships hold on $\mathcal{L}$.
\end{abstract}

KEY WORDS AND PHRASES. Lindelöf lattice, 0-1 valued measures, disjunctive lattice, countably compact, normal lattice, prime complete, premeasure, delta lattice, replete, regular, slightly normal, I-lattice.

1980 AMS SUBJECT CLASSIFICATION CODE. 28A60, 28 A52.

1. INTRODUCTION. In this paper we consider in detail, Lindelöf lattices and their relationship to separation properties, and also to certain measure properties. In particular, we show that the generalized Wallman replete space is a Lindelöf space if and only if a certain measure relationship holds on $\mathcal{L}$, and, similarly, the generalized Wallman prime complete space is Lindelö if and only if another measure relationship holds on $\mathcal{L}$.

Before doing this, we investigate some general properties of regular, Lindelöf lattices as well as normal and slightly normal lattices. We are mainly concerned with measure implications of these properties, and also lattice separation properties that may result.

We adhere to standard lattice and measure terminology and notation (See e.g. [1], [2], [3], and [7]), and we give some relevant background material in Section 2 for the reader's convenience.

2. BACKGROUND AND NOTATION. Let $X$ be an abstract set and $\mathcal{L}$ a lattice of subsets of $X$. It is assumed that $\emptyset$ and $X \in \mathcal{L}$, although this is not necessary for some of our results.

MEASURE TERMINOLOGY. We now introduce some measure terminology and results.

1. $a(\mathcal{L})$ is the algebra generated by $\mathcal{L}$.

2. $I(\ell)$ is the set of all $0-1$ valued finitely additive measures defined on a $(\ell)$. Also, we note that there exists a $1-1$ correspondence between $\mu \in I(\ell)$ and prime $\ell$-filters given by $F=\{\in \ell$; $\mu(L)=1$ \} .

3. $I_{R}(\mathcal{L})$ is the set of all $\mu \in I(\mathcal{L})$ which are $\mathcal{\ell}$-regular; that is, for $A \in a(\mathcal{L}), \mu(A)=\sup \mu(L)$ where $L \subset A, L \in \mathcal{L}$. Observe that the correspondence given in 2 gives a $1-1$ correspondence between $I_{R}(\mathcal{L})$ and $\mathcal{L}$-ultrafilters. 
4. $I_{\sigma}(\mathcal{L})$ is the set of all 0-1 $\sigma$-smooth finitely additive measures on $\mathcal{L}$. $\quad \mu \in I(\mathcal{L})$ is $\sigma$-smooth if for $L_{n} \in \mathcal{L}, L_{n} \downarrow \emptyset$ then $\lim _{n \rightarrow \infty} \mu\left(L_{n}\right)=0$. Also, we note that $\mu \in I_{\sigma}(\mathcal{L})$ if and only if the associated prime $\mathcal{\ell}$-filter given in 2 has the countable intersection property (c.i.p.).

5. $I^{\sigma}(\mathcal{L})$ is the set of all 0-1 $\sigma$-smooth finitely additive measures on a ( $\left.\mathcal{L}\right)$. A $0-1$ measure $\mu$ is $\sigma$-smooth on a $(\mathcal{L})$ if for $A_{n} \in \mathcal{L}, A_{n} \downarrow \emptyset$, then $\lim _{n \rightarrow \infty} \mu\left(A_{n}\right)=0$.

6. It is worthwhile noting that if $\mu \in I_{R}(\mathcal{L})$ then $\mu \in I^{\sigma}(\mathcal{L})$ if and only if $\mu \in I_{\sigma}(\mathcal{L})$.

7. $I_{R}^{\sigma}(\mathcal{L})$ is the set of all $\mathcal{L}$-regular measures of $I^{\sigma}(\mathcal{L})$.

8. $\Pi(\mathcal{L})$ is the set of all premeasures on $\mathcal{L}$; i.e., $\pi \in \Pi(\mathcal{L})$ if $\pi: \mathcal{L} \rightarrow\{0,1\}$ and $\pi(\emptyset)=0, \pi$ is monotone and $\pi(A)=\pi(B)=1$ for $A, B \in \mathcal{L}$ then $\pi(A \cap B)=1$. Also, it is easy to see that there exists a $1-1$ correspondence between $\mathcal{L}$ filters and premeasures on $\mathcal{L}$. In addition, if $\pi \in \Pi(\mathcal{L})$ and $L_{n} \downarrow \emptyset, L_{n} \in \mathcal{L}$ then $\lim _{n \rightarrow \infty} \pi\left(L_{n}\right)=0$ then we write $\pi \in \Pi_{\sigma}(\mathcal{L})$.

9. For $\mu \in I(\ell)$, we define the support of $\mu$ to be $S(\mu)=\{\cap L ; L \in \mathcal{L} ; \mu(L)=1\}$. Similarly, we define $S(\pi)$ for $\pi \in \Pi(\ell)$.

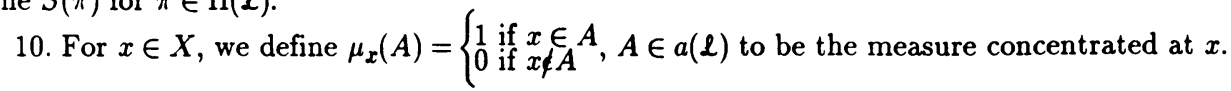

11. By $\mu \leq \nu(\mathcal{L})$, we simply mean $\mu(L) \leq \nu(L)$ for all $L \in \mathcal{L}$.

LATTICE TERMINOLOGY. We next present some lattice terminology and results.

$1^{\prime} . \quad \delta(\mathcal{L})$ is the lattice of all countable intersections of sets from $\mathcal{L}$.

$2^{\prime} . \quad \tau(\mathcal{L})$ is the lattice of arbitrary intersections of sets from $\mathcal{L}$.

$3^{\prime}$. $\mathcal{L}^{\prime}$ is the complementary lattice of $\mathcal{L}$; i.e., $\mathcal{L}^{\prime}=\left\{L^{\prime} ; L \in \mathcal{L}\right\}$, where prime denotes complement.

4'. $\mathcal{L}$ is disjunctive if for any $x \in X$ and $L_{1} \in \mathcal{L} ; x \notin L_{1}$, there exists an $L_{2} \in \mathcal{L} ; x \in L_{2}^{\prime}$ and $L_{1} \cap L_{2}=\emptyset$. Also, we note that $\mathcal{L}$ is disjunctive if and only if $\mu_{x} \in I_{R}(\mathcal{L})$ for all $x \in X$.

5'. $\mathcal{L}$ is regular if for any $x \in X$ and $L_{1} \in \mathcal{L} ; x \notin L_{1}$, there exist $L_{2}, L_{3} \in \mathcal{L} ; x \in L_{2}^{\prime}, L_{1} \subset L_{3}^{\prime}$ and $L_{2}^{\prime} \cap L_{3}^{\prime}=\emptyset$. Also, $\ell$ is regular if and only if $\mu_{1} \leq \mu_{2}(\ell), \mu_{1}, \mu_{2} \in I(\mathcal{L})$ implies $S\left(\mu_{2}\right) \tau \mathrm{s}\left(\mu_{1}\right)$.

$6^{\prime} . \quad \mathcal{L}$ is normal if for any $L_{1}, L_{2} \in \mathcal{L} ; L_{1} \cap L_{2}=\emptyset$, there exist $L_{3}, L_{4} \in \mathcal{L} ; L_{1} \subset L_{3}^{\prime}, L_{2} \subset L_{4}^{\prime}$ and $L_{3}^{\prime} \cap L_{4}^{\prime}=\emptyset$. Also, $\mathcal{L}$ is normal if and only if for any $\mu \in I(\ell)$ there exists a unique $\nu \in I_{R}(\mathcal{L})$ such that $\mu \leq \nu(\mathcal{L})$.

$7^{\prime}$. Let $\ell_{1}$ and $\ell_{2}$ be two lattices of subsets of $X . \quad \ell_{1}$ separates $\ell_{2}$ if $L_{1}, L_{2} \in \ell_{2} ; L_{1} \cap L_{2}=0$ then, there exist $L_{3}, L_{4} \in L_{1} ; L_{1} \subset L_{3}, L_{2} \subset L_{4}$ and $L_{3} \cap L_{4}=\emptyset$.

$8^{\prime} . \quad \mathcal{L}$ is Lindelöf if $\bigcap_{x} L_{x}=\emptyset$ where $L \in \mathcal{L}$ then, for a countable subcollection $L_{x}, i=1,2, \cdots$ of $L, \bigcap_{i=1}^{\infty} L_{x_{i}}=\emptyset$. Equivalently, $\mathcal{L}$ is Lindelöf if and only if for each $\pi \in \Pi_{\sigma}(\mathcal{L}), S(\pi) \neq \emptyset^{i}$.

$9^{\prime} . \quad \mathcal{L}$ is countably compact if every countable covering of $X$ by elements of $\mathcal{L}^{\prime}$ has a finite subcovering.

$10^{\prime} . \mathcal{L}$ is replete if for any $\mu \in I_{R}^{\sigma}(\mathcal{L}), S(\mu) \neq \emptyset$.

$11^{\prime} . \mathcal{L}$ is a delta lattice ( $\delta$-lattice) if $\delta(\mathcal{L})=\mathcal{L}$.

Further related material can be found in ( [4\}, [5], [6] and [8]).

3. ON SEPARATION. This section begins with the following observations which follows directly from the definitions.

(1) $\mathcal{L}$ is Lindelöf if and only if $\tau \mathcal{L}$ is Lindelöf; and

(2) if $\mathcal{L}$ is $\delta$ and Lindelöf then, $\mathcal{L}$ separates $\tau \mathcal{L}$.

Now we investigate some implications of lattices properties for the correspondent measures.

THEOREM 3.1. If $\mathcal{L}$ is regular and Lindelöf then, $I_{\sigma}\left(\mathcal{L}^{\prime}\right) \subset I_{\sigma}(\mathcal{L})$.

PROOF. Suppose $I_{\sigma}\left(\mathcal{L}^{\prime}\right) \not \subset I_{\sigma}(\mathcal{L})$; that is, there exists a $\mu \in I_{\sigma}\left(\mathcal{L}^{\prime}\right)$ but not in $I_{\sigma}(\mathcal{L})$. Let $\rho(L)=\sup \mu\left(\hat{L}^{\prime}\right), \hat{L}^{\prime} \subset L, \widehat{L}^{\prime}, L \in \mathcal{L}$ which is a premeasure; that is, $\rho \in \Pi(\mathcal{L})$. But since $\mathcal{L}$ is regular then $S(\mu)=S(\rho)$. Also, $\rho \in \Pi_{\sigma}(\mathcal{L})$ since $\mu \in I_{\sigma}\left(\mathcal{L}^{\prime}\right)$, and since $\mathcal{L}$ is Lindelöf, $S(\rho) \neq \emptyset$ then $S(\mu) \neq \emptyset$. 
Let $x \in S(\mu)$, then $\mu \leq \mu_{x}(\mathcal{L})$ therefore $\mu \in I_{\sigma}(\mathcal{L})$ this is a contradiction. Thus, $I_{\sigma}\left(\mathcal{L}^{\prime}\right) \subset I_{\sigma}(\mathcal{L})$.

DEFINITION 3.1. $\quad \mathcal{L}$ is slightly normal if for every $\mu \in I_{\sigma}\left(\ell^{\prime}\right)$, there exists a unique $\nu \in I_{R}(\mathcal{L}) ; \mu \leq \nu(\mathcal{L})$.

THEOREM 3.2. a) If $\delta\left(\mathcal{L}^{\prime}\right)$ separates $\mathcal{L}$, then $\mathcal{L}$ is slightly normal; b) Suppose whenever $A \cap B=\emptyset, A, B \in \mathcal{L}$, there exist $\hat{A}, B_{n} \in \mathcal{L}$ such that $A \subset \widehat{A} \subset \bigcup_{n=1}^{\infty} B_{n} \subset B^{\prime}$ then $\delta\left(\mathcal{L}^{\prime}\right)$ separates $\mathcal{L}$.

PROOF. Let $\mu \in I_{\sigma}\left(\mathcal{L}^{\prime}\right), \mu \leq \nu_{1}(\mathcal{L}), \mu \leq \nu_{2}(\mathcal{L})$ where $\nu_{1}, \nu_{2} \in I_{R}(\mathcal{L})$. If $\nu_{1} \neq \nu_{2}$, there exist $A, B \in \mathcal{L}, \nu_{1}(A)=\nu_{2}(B)=1$ and $\nu_{2}(A)=\nu_{1}(B)=0$ then by hypothesis, there exist $L_{n}^{\prime}, L_{m}^{\prime}$ where $L_{n}, L_{m} \in \mathcal{L}$ for all $n, m$ such that $A \subset \cap L_{n}^{\prime}, B \subset \cap L_{m}^{\prime}$ and $\left(\cap L_{n}^{\prime}\right) \cap\left(\cap L_{m}^{\prime}\right)=\emptyset$. Since $\nu_{1}(A)=1$ and $\mu \leq \nu_{1}(\mathcal{L})$, then $\mu\left(L_{n}^{\prime}=1\right)$ for all $n$. Similarly, we get $\mu\left(L_{m}^{\prime}\right)=1$ for all $m$. Thus, $\mu\left(L_{n}^{\prime} \cap L_{m}^{\prime}=1\right.$ for all $n, m$ but ${ }_{n, m} L_{n}^{\prime} \cap L_{m}^{\prime}=\emptyset$ which is a contradiction since $\mu \in I_{\sigma}\left(\ell^{\prime}\right)$. Thus, $\nu_{1} \leq \nu_{2}$ and so $\mathcal{L}$ is slightly normal; $\mathrm{c}$ ) the proof is clear.

Next, we consider the following condition, designated as (*)

Let $A, E \in \mathcal{L} ; A \cap B=\emptyset$ then, there exists $A_{i}^{\prime} \uparrow$ and $B_{i} \in \mathcal{L} ; A \subset \bigcup_{i=1}^{\infty} A_{i}^{\prime}$, $A_{i} \in \mathcal{L}$ and $A_{i}^{\prime} \subset B_{i}$ and also $B_{i} \cap B=\emptyset$ for all $i$.

THEOREM 3.3. If $\mathcal{L}$ is $\delta$ and satisfies $(*)$ then, $\mathcal{L}$ is normal.

PROOF. Let $A \cap B=\emptyset$ for $A, B \in \mathcal{L}$ then, there exists $C_{i}^{\prime} \uparrow, C_{i} \in \mathcal{L} ; B \subset_{i} \bigcup_{=1}^{\infty} C_{i}^{\prime}$ and there exists $D_{i} \in \mathcal{L} ; C_{i}^{\prime} \subset D_{i}$ and $D_{i} \cap A=\emptyset$ for all $i$. Let $S_{i}=A_{i}^{\prime} \cap D_{i}^{\prime}$ and $H_{j}=C_{j}^{\prime} \cap B_{j}^{\prime}$ clearly $S_{i}, H_{j} \in \mathcal{L}^{\prime}$. Then, $S_{i} \cap H_{j}=\emptyset$ since $S_{i} \cap H_{j}=A_{i}^{\prime} \cap D_{i}^{\prime} \cap C_{j}^{\prime} \cap B_{j}^{\prime}$ and $i \leq j$ then clearly $A_{i}^{\prime} \subset A_{j}^{\prime} \subset B_{j}$, while if $i>j$ then clearly $C_{j}^{\prime} \subset C_{i}^{\prime} \subset D_{i}$. Also, $\underset{i}{\cup} S_{i}=\bigcup_{i}\left(A_{i}^{\prime} \cap D_{i}^{\prime}\right) \supset A$ since $\bigcup_{i} A_{i}^{\prime} \subset A$ and $D_{i}^{\prime} \subset A$ for all $f$. Similarly, $\underset{j}{\cup} H_{j} \supset B$ and $\bigcup_{i} S_{i}, \underset{j}{\cup} H_{j} \in \mathcal{L}^{\prime}$ if $\mathcal{L}^{i}$ is $\delta$ and so they are disjoint as shown. Thus, $\mathcal{L}$ is normal.

THEOREM 3.4. If $\mathcal{L}$ is $\delta$, regular and Lindelöf then, $\mathcal{L}$ is normal.

PROOF. Let $A, B \in \mathcal{L}$ and $A \cap B=\emptyset$. Since $\mathcal{L}$ is regular, for $x \in A$ there exist $L_{x}, \widehat{L}_{x} \in \mathcal{L}$ such that $x \in L_{x}^{\prime} \subset \widehat{L}_{x}$, and $\widehat{L}_{x} \cap B=\emptyset$. Since $\mathcal{L}$ is Lindelöf there exists a countable number $L_{x_{i}}, i=1,2, \cdots$ of the $L_{x}$ such that $A \subset \cup L_{x}^{\prime}$, and we may assume $L_{x_{i}}^{\prime} \uparrow$. Then, $L_{x_{i}}^{\prime} \subset \hat{L}_{x_{i}}$ and $\hat{L}_{x_{i}} \cup B=\emptyset$ so condition (*) is satisfied and so since $\mathcal{L}$ is $\delta$, it follows that $\mathcal{L}$ is normal by Theorem $3.3^{i}$.

Next, for $\mu \in I(\mathcal{L})$ and $E \subset X$, we define $\mu^{\prime}(E)=\inf \mu\left(L^{\prime}\right)$ where $E \subset L^{\prime}, L \in \mathcal{L}$. And, we say that $\mu \in I_{w}(\mathcal{L})$ if $\mu\left(L^{\prime}\right)=1, L \in \mathcal{L}$ then $L^{\prime} \supset \widehat{L} \in \mathcal{L}$ and $u^{\prime}(\widehat{L})=1$. Also, we note that $\mu=\mu^{\prime}(\mathcal{L})$ if and only if $\mu \in I_{R}(\mathcal{L})$.

THEOREM 3.5. If $\mathcal{L}$ is normal then, $I_{w}(\mathcal{L})=I_{R}(\mathcal{L})$.

PROOF. i) If $\mu \in I_{R}(\mathcal{L})$ and $\mu\left(L^{\prime}\right)=1, L \in \mathcal{L}$ then $L^{\prime} \supset \hat{L} \in \mathcal{L}, \mu(\widehat{L})=1$ but $\mu \leq \mu^{\prime}(\mathcal{L})$ therefore $\mu^{\prime}(\hat{L})=1$. Thus, $\mu \in I_{w}(\mathcal{L})$ and so $I_{R}(\ell) \subset I_{w}(\mathcal{L})$; ii) In general, we know that $\mu \leq \mu^{\prime}(\ell)$. Now, suppose $\mathcal{L}$ is normal and $\mu \in I_{w}(\mathcal{L})$. Suppose $\mu(L)=0, L \in \mathcal{L}$ then $\mu\left(L^{\prime}\right)=1$ therefore $L^{\prime} \supset \hat{L} \in \mathcal{L}$ and $\mu^{\prime}(\widehat{L})=1$. By normality $L \subset A^{\prime}, \widehat{L} \subset B^{\prime}, A, B \in \mathcal{L}$ and $A^{\prime} \cap B^{\prime}=\emptyset$. Since $\mu^{\prime}(\widehat{L})=1$, therefore $\mu\left(B^{\prime}\right)=1$ and hence $\mu^{\prime}(L)=0$.

One may easily note that the converse of Theorem 3.5 is not true.

THEOREM 3.6. If $\mu \in I_{\sigma}\left(\mathcal{L}^{\prime}\right) \cap I_{w}(\mathcal{L})$ and $\delta\left(\mathcal{L}^{\prime}\right)$ separates $\mathcal{L}$ then, $\mu \in I_{R}(\mathcal{l})$.

PROOF. Let $L_{1} \in \mathcal{L}$ and $\mu\left(L_{1}\right)=0$ so $\mu\left(L_{1}^{\prime}\right)=1$ then $L_{1}^{\prime} \supset L_{2} \in \mathcal{L}$ with $\mu^{\prime}\left(L_{2}\right)=1$ since $\mu \in I_{w}(\mathcal{L})$. Since $\delta\left(\mathcal{L}^{\prime}\right)$ separates $\mathcal{L}, L_{1} \subset \cap A_{n}^{\prime}, A_{n} \in \mathcal{L}, L_{2} \subset \cap B_{m}^{\prime}, B_{m} \in \mathcal{L}$ and $\left(\cap A_{n}^{\prime}\right) \cap$ $\left(\cap B_{m}^{\prime}\right)=\emptyset$ so $_{n, m} A_{m}^{\prime} \cap B_{m}^{\prime}=\emptyset$ (and we may assume $\downarrow$ ). Therefore, $\mu\left(A_{n}^{\prime} \cap B_{m}^{\prime}\right)=0$ for $n, m$ big since $\mu \in I_{\sigma}\left(\mathcal{L}^{\prime}\right)$ but $L_{2} \subset B_{m}^{\prime}$ therefore $1=\mu^{\prime}\left(L_{2}\right) \leq \mu^{\prime}\left(B_{m}^{\prime}\right)=\mu\left(B_{m}^{\prime}\right)$ since in general $\mu=\mu^{\prime}\left(\ell^{\prime}\right)$ 
therefore $\mu\left(A_{n}^{\prime}\right)=0$ for $n$ big but $L_{1} \subset A_{n}^{\prime}$ then $L_{1}^{\prime} \supset A_{n}$ and $\mu\left(A_{n}\right)=1$. Thus, $\mu \in I_{R}(\mathcal{l})$.

4. LINDELÖF LATTICES. This section is divided into two parts.

PART A

DEFINITION 4.1. $\mathcal{L}$ is an $I$-lattice if for every $\pi \in \Pi_{\sigma}(\mathcal{L})$, there exists a $\mu \in I_{R}^{\sigma}(\mathcal{L}) ; \pi \leq \mu(\mathcal{L})$.

THEOREM 4.1. If $\mathcal{L}$ is an $I$-lattice and replete then, $\mathcal{L}$ is Lindelöf.

PROOF. Let $\pi \in \Pi_{\sigma}(\mathcal{L})$ then there exists a $\mu \in I_{R}^{\sigma}(\mathcal{L}) ; \pi \leq \mu(\mathcal{L})$ since $\mathcal{L}$ is an $I$-lattice. Since $\mathcal{L}$ is replete $S(\mu)=\cap L \neq \emptyset$ where $\mu(L)=1, L \in \mathcal{L}$. In addition, $S(\pi) \subset S(\mu)$ where $S(\pi)=\cap L_{x}$ and $\pi\left(L_{x}\right)=1, L_{x} \in \mathcal{L}$ for all $x$. Thus, $S(\pi) \neq \emptyset$ and so $\mathcal{L}$ is Lindelöf.

THEOREM 4.2. If $\ell$ is countably compact then, $\ell$ is an $I$-lattice.

PROOF. Let $\pi \in \Pi_{\sigma}(\mathcal{L})$ then there exists a $\mu \in I_{R}(\mathcal{L}) ; \pi \leq \mu(\mathcal{L})$ but since $\mathcal{L}$ is countably compact $\mu \in I_{R}^{\sigma}(\mathcal{L})=I_{R}(\mathcal{L})$ and so $\mathcal{L}$ is an $I$-lattice.

THEOREM 4.3. If $\mathcal{L}$ is disjunctive and Lindelöf then, $\ell$ is an $I$-lattice.

PROOF. Let $\pi \in \Pi_{\sigma}(\mathcal{L})$. Since $\mathcal{L}$ is Lindelöf then $S(\pi) \neq \emptyset$. Let $x \in S(\pi)$ since $\mathcal{L}$ is disjunctive, $\mu_{x} \in I_{R}(\mathcal{L})$ where $\pi \leq \mu_{x}(\mathcal{L})$ and $\mu_{x}$ is $\sigma$-smooth on $\mathcal{L}$ then $\mu_{\mathrm{x}} \in I_{R}^{\sigma}(\mathcal{L})$ and so $\mathcal{L}$ is an $I$ lattice.

Next, we consider $W_{\sigma}(\mathcal{L})=\left\{W_{\sigma}(L) ; L \in \mathcal{L}\right\}$ where $W_{\sigma}(L)=\left\{\mu \in I_{R}^{\sigma}(\mathcal{L}) ; \mu(L)=1\right\}$. Note that $W_{\sigma}(\ell)$ forms a base for the closed sets $\tau W_{\sigma}(\ell)$ of $I_{R}^{\sigma}(\ell)$. Also, we may note the following wellknown fact:

1) If $\mathcal{L}$ is disjunctive then, $I_{R}^{\sigma}(\mathcal{L}), W_{\sigma}(\mathcal{L})$ is replete.

THEOREM 4.4. If $\mathcal{L}$ is disjunctive and an $I$-lattice then, the topological space $I_{R}^{\sigma}(\mathcal{L}), \tau W_{\sigma}(\mathcal{L})$ is Lindelöf.

PROOF. Since $\mathcal{L}$ satisfies conditions for an $I$-lattice so does $W_{\sigma}(\mathcal{L})$. Also, since $\mathcal{L}$ is disjunctive then, $W_{\sigma}(\ell)$ is replete. Thus, by Theorem $4.1, W_{\sigma}(\mathcal{L})$ is Lindelöf and so is $\tau W_{\sigma}(\ell)$.

THEOREM 4.5. Assume $\mathcal{L}$ is disjunctive. If $I_{R}^{\sigma}(\mathcal{L}), \tau W_{\sigma}(\mathcal{L})$ is Lindelöf, then $\mathcal{L}$ is an $I$-lattice.

PROOF. Let $\mu \in I_{R}^{\sigma}(\mathcal{L})$ and $\mu \notin W_{\sigma}(L), L \in \mathcal{L}$ then, $\mu\left(L^{\prime}\right)=1$ therefore $L^{\prime} \supset \hat{L} \in \mathcal{L}$ and $\mu(\hat{L})=1$ therefore $\mu \in W_{\sigma}(\hat{L})$ and $W_{\sigma}(\hat{L}) \cap W_{\sigma}(L)=\emptyset$ therefore $W_{\sigma}(\mathcal{L})$ is disjunctive. Also, $W_{\sigma}(\mathcal{L})$ is Lindelöf then, by Theorem $4.3, W_{\sigma}(\mathcal{L})$ is an $I$-lattice. Thus, $\mathcal{L}$ is an $I$-lattice.

\section{PART B}

We now consider the following condition, designated as $(* *)$ [For every $\pi \in \Pi_{\sigma}(\mathcal{L})$, there exists a $\left.\nu \in I_{\sigma}(\mathcal{L}) ; \pi \leq \nu(\mathcal{L})\right](* *)$.

DEFINITION 4.2. $\mathcal{L}$ is a prime complete if for $\mu \in I_{\sigma}(\mathcal{L}), S(\mu) \neq \emptyset$.

THEOREM 4.6. If $\mathcal{L}$ is prime complete and satisfies $(* *)$ then, $\mathcal{L}$ is Lindelöf.

PROOF. If $\mathcal{L}$ satisfies $(* *)$ then, for $\pi \in \Pi_{\sigma}(\mathcal{L})$ there exists a $\nu \in I_{\sigma}(\mathcal{L}) \pi \leq \nu(\mathcal{L})$. By prime completeness of $\mathcal{L}, S(\nu) \neq \emptyset$ but $S(\nu) \subset S(\pi)$ then $S(\pi) \neq \emptyset$ and so $\mathcal{L}$ is Lindelöf.

THEOREM 4.7. If $\mathcal{L}$ is countably compact then, $(* *)$ is satisfied.

PROOF. Let $\pi \in \Pi_{\sigma}(\mathcal{L})$ then there exists a $\nu \in I(\mathcal{L}) \subset I_{\sigma}(\mathcal{L})$ since $\mathcal{L}$ is countably compact; $\pi \leq \nu(\ell)$ and so $(* *)$ is satisfied.

THEOREM 4.8. If $\ell$ is Lindelöf then, $(* *)$ is satisfied.

PROOF. Let $\pi \in \Pi_{\sigma}(\ell): S(\pi) \neq \emptyset$ since $\ell$ is Lindelöf. Let $x \in S(\pi)$ then, there exists a $\nu=\mu_{x} \in I_{\sigma}(\mathcal{L})$ and $\pi \leq \mu_{x}(\mathcal{L})$ and so $(* *)$ is satisfied.

Consider the space $I_{\sigma}(\mathcal{L})$ and the lattice $V_{\sigma}(\mathcal{L})$ where $V_{\sigma}(\mathcal{L})=\left\{V_{\sigma}(L) ; L \in \mathcal{L}\right\}$ and $V_{\sigma}(L)=\left\{\mu \in I_{\sigma}(\mathcal{L}) ; \mu(L)=1\right\}$. It is well-known that $V_{\sigma}(\mathcal{l})$ is prime complete (See [1] and $V_{\sigma}(\mathcal{L})$ is a base for the closed sets $\tau V_{\sigma}(\mathcal{L})$ of $I_{\sigma}(\mathcal{L})$.

THEOREM 4.9. $\mathcal{L}$ satisfies $(* *)$ if and only if the topological space $I_{\sigma}(\mathcal{L}), \tau V_{\sigma}(\mathcal{L})$ is Lindelöf.

PROOF. i) If $I_{\sigma}(\mathcal{L}), \tau V_{\sigma}(\ell)$ is Lindelöf then, $V_{\sigma}(\mathcal{L})$ is Lindelöf and by Theorem $4.8, V_{\sigma}(\mathcal{L})$ satisfies (**) and so does $\mathcal{L}$; ii) Assume that $\mathcal{L}$ satisfies $(* *)$ then, $V_{\sigma}(\mathcal{L})$ satisfies $(* *)$. Moreover, 
$V_{\sigma}(\mathcal{L})$ is prime complete. Thus, by Theorem 4.6, $I_{\sigma}(\mathcal{L}), V_{\sigma}(\mathcal{L})$ is Lindelöf which implies that $I_{\sigma}(\ell), \tau V_{\sigma}(\ell)$ is Lindelöf.

\section{REFERENCES}

1. BACHMAN, G. and STRATIGOS, P., On general lattice repleteness and completeness, lll. J. Math., 27(4), (1983), pp. 535-561.

2. regular measures with applications, Can. J. Math., 33 (1981), pp. 1498-1525.

3. FROLIK, Z., Prime filters with the C.I.P., Comm. Math. Univ. Carolinae, 13 (1972), pp. 533 575.

4. GRASSI, P., On subspaces of replete and measure replete spaces, Can. Math. Bull., 27(1), (1984), pp. 58-64.

j. HUERTA, C., Measure requirements on distributive lattices for Boolean algebras and topological applications, Proc. Amer. Math. Soc.,_2(1989), pp. 307-309.

6. HUERTA, C., Notions of compactness on the lattice and the point set in terms of measures, Ann. sc. math. Québec, 13 (1), 1989, pp. 49-52.

7. NOEBELING, G., Grundlagen der analytischen topologie, Springer-Verlag, Berlin 1954.

8. SZETO, M., Measure repleteness and mapping preservations, J. Indian. Math. Soc., 43(1979), pp. 35-52. 


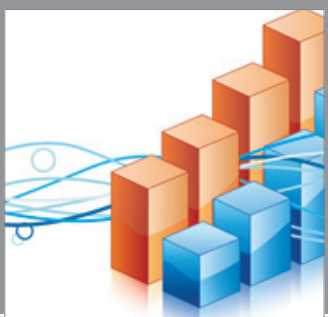

Advances in

Operations Research

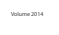

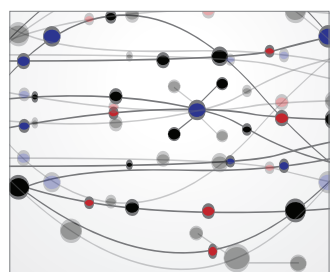

\section{The Scientific} World Journal
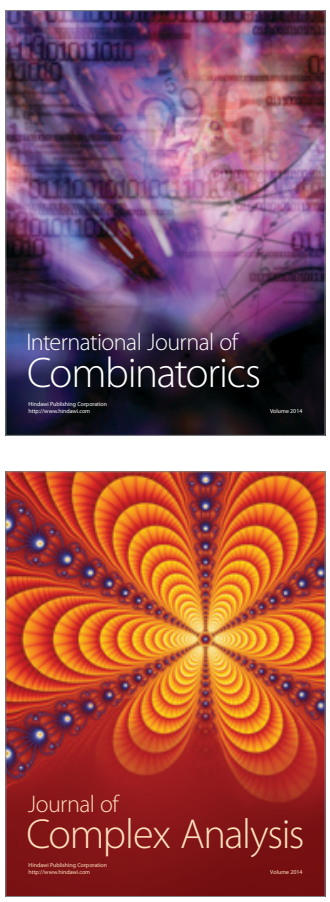

International Journal of

Mathematics and

Mathematical

Sciences
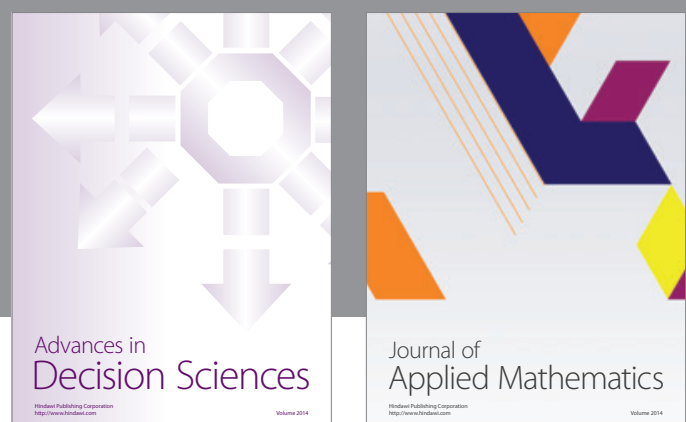

Journal of

Applied Mathematics
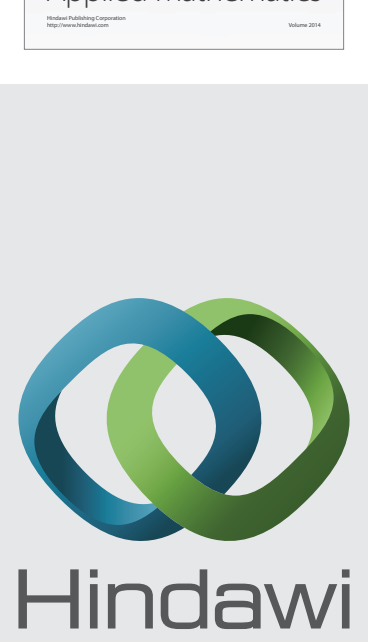

Submit your manuscripts at http://www.hindawi.com
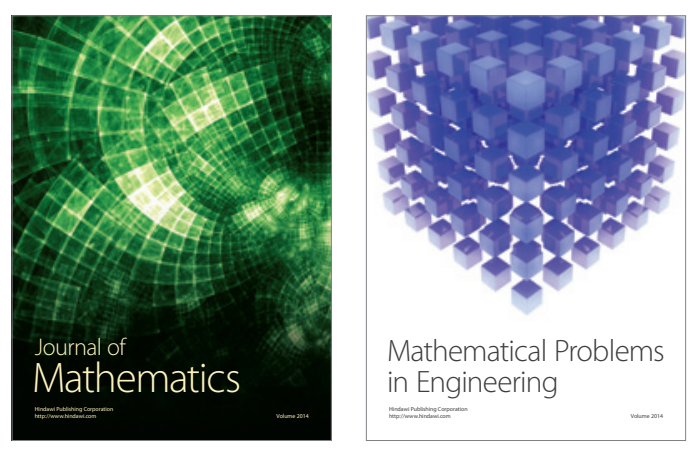

Mathematical Problems in Engineering
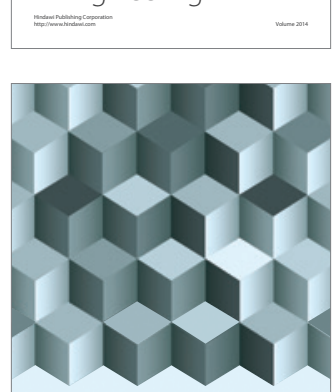

Journal of

Function Spaces
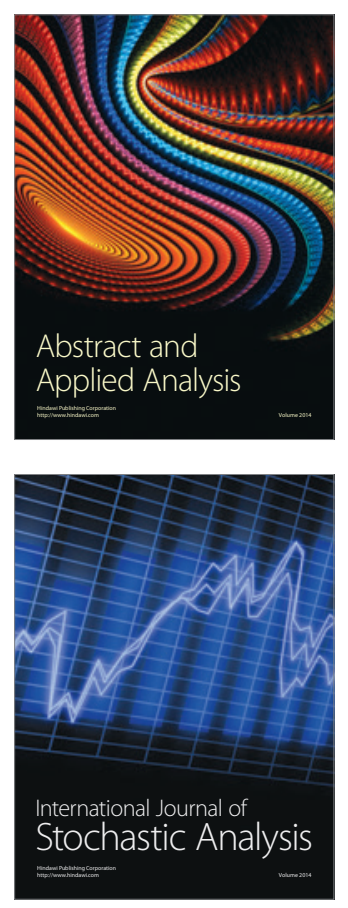

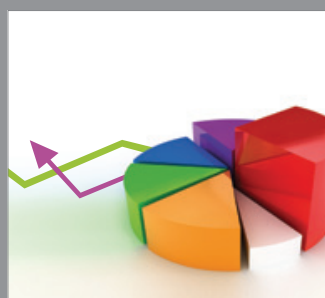

ournal of

Probability and Statistics

Promensencen
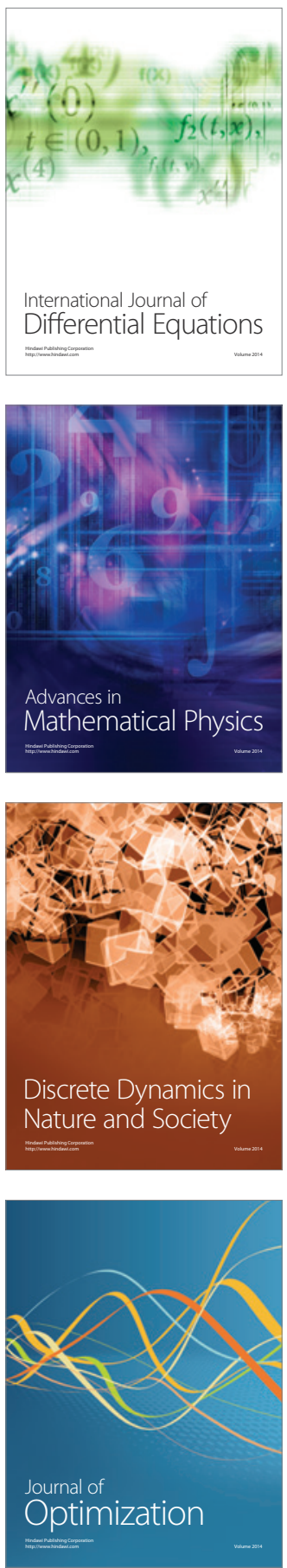